\title{
FAR FIELD SCATTERING FROM BODIES OF REVOLUTION *)
}

\author{
by $\mathrm{K}$. M. SIEGEL
}

The University of Michigan Ann Arbor, Michigan, U.S.A.

\section{Summary}

By use of approximations based on physical reasoning radar cross-section results for bodies of revolution are found. In the Rayleigh region (wavelength large with respect to object dimensions) approximate solutions are found. Examples given include a finite cone, a lens, an elliptic ogive, a spindle and a finite cylinder. In the physical optics region (wavelength very small with respect to all radii of curvature) Kirchhoff theory and also geometric optics can be used. When the body dimensions are only moderately large with respect to the wavelength, Fock or Franz theory can be applied, and examples of the circular and elliptic cylinder are presented. In the region where some dimensions of the body are large with respect to the wavelength and other dimensions are small with respect to the wavelength, special techniques are used. One example, the finite cone, is solved by appropriate use of the wedgelike fields locally at the base. Another example is the use of traveling wave theory for obtaining approximate solutions for the prolate spheroid and the ogive. Other results are obtained for cones the base perimeter of which is of the order of a wavelength by using known results for rings of the same perimeter.

§1. Introduction. It is the intent in this paper to use different mathematical techniques to obtain approximate results for the far zone scattering of plane electromagnetic waves by perfectly conducting bodies of revolution for all ratios of body dimension to wavelength. In many places speculation based on physical reasoning has replaced mathematical-rigour. We shall first discuss the Rayleigh region, then the physical optics region and then the resonance region.

\section{§2. Rayleigh cross-section of bodies of revolution. Rayleigh}

*) This paper with minor revision is as the author presented it at the URSI XIIth General Assembly in Boulder, Colorado August 22-September 5, 1957. 
scattering ${ }^{1}$ ) describes the scattering of electromagnetic radiation by a body whose dimensions are much smaller than the wavelength of the radiation. Thus the Rayleigh limit describes the scattered field, due to an incident plane wave, approximated at a large distance from the body by the field of radiating electric and magnetic dipoles located at the scatterer (the magnetic dipole contribution is comparable to that of the electric dipole only for a perfect conductor). To evaluate the electric (magnetic) dipole moment, the static electric (magnetic) field induced on the body by an applied constant field must be known. In other words, the electrodynamic boundary-value problem has been reduced to a corresponding static problem.

Although the solution of the Laplace equation is in principle simpler than the solution of the Maxwell equations, there are very few geometrical cases for which even the former is manageable. The question, therefore, arises whether any approximate information can be obtained as to the Rayleigh cross-section when a solution of the Laplace equation is not available. That this should be possible is heuristically plausible. When the wavelength is much longer than the dimensions of a body, one cannot discern details of the structure of the body: the observed effect depends more on the size of the body than on its shape. Thus, knowledge of the size of the body. modified by a rough indication of shape, should suffice for a description of the body in finding the Rayleigh cross-section. It is the purpose of the present discussion to explore this possibility.

As background, it might be helpful to bear in mind a couple of features of the Rayleigh approximation itself. The solution to an electromagnetic scattering problem can be expressed as a multipole expansion. The relative importance of terms in the expansion depends upon the distance of the observer from the scatterer (as well as on the dimensions of the body relative to the wavelength), so that a small error in describing the field in one region can result in completely misrepresenting the corresponding field elsewhere. For a scatterer much smaller than the wavelength, retaining only the dipole terms gives a good approximation to the far zone, though the field in the near zone may be entirely wrong. Specifying the dipole moments of the body does not determine the body uniquely (i.e. different bodies may have the same dipole moments). Thus the Rayleigh cross-section alone cannot identify the body fully. On the 
other hand, the finer details of the structure of the body, which would be exhibited by the higher moments (and seriously affect the cross-section at small wavelengths), do not affect the Rayleigh cross-section.

For simplicity, consider the scatterer to be a body of revolution, make it a perfect conductor. (this is a rather trivial limitation) and examine back-scattering of a plane wave incident along the axis of symmetry (there is then no polarization dependence). Thus, the direction of incidence will be denoted by $z$, the incident electric vector direction by $x$, the incident magnetic vector direction by $y$ and the length of the body along the symmetry axis by $l$. The electric dipole moment $\boldsymbol{p}$ is given by

$$
\boldsymbol{p}=\int_{S} \omega \boldsymbol{r} \mathrm{d} S
$$

where $\omega$ is the charge density, $\boldsymbol{r}$ the position vector and $S$ the surface of the body. The boundary condition yields.

$$
\omega=\varepsilon \boldsymbol{E} \cdot \boldsymbol{n}=\varepsilon E,
$$

where $\varepsilon=$ dielectric constant, $\boldsymbol{n}=$ unit outward normal to the surface and $\boldsymbol{E}=$ electric field strength. Using cylindrical coordinates,

$$
\mathrm{d} S=\rho \sqrt{1+(\mathrm{d} \rho / \mathrm{d} z)^{2}} \mathrm{~d} \phi \mathrm{d} z,
$$

where $\rho$ is a function of $z$ but not of $\dot{\phi}$, so that

$$
\boldsymbol{p}=\varepsilon \int_{0}^{l} \mathrm{~d} z \rho \sqrt{1+\left(\frac{\mathrm{d} \rho}{\mathrm{d} z}\right)^{2}} \int_{0}^{2 \pi} \mathrm{d} \phi E \boldsymbol{r} .
$$

From uniqueness and symmetry considerations we can write

$$
E=\sum_{n=0}^{\infty} \dot{a}_{n}(z) \cos n \phi
$$

Then $p_{y}=0, p_{z}=0$ and

$$
\begin{aligned}
p_{x} & =\varepsilon \int_{0}^{l} \mathrm{~d} z \rho^{2} \sqrt{1+\left(\frac{\mathrm{d} \rho}{\mathrm{d} z}\right)^{2}} \int_{0}^{2 \pi} \mathrm{d} \phi \cos \phi\left[\sum_{n=0}^{\infty} a_{n}(z) \cos n \phi\right] \\
& =\varepsilon \int_{0}^{l} \pi \mathrm{d} z \rho^{2} a_{1}(z) \sqrt{1+\left(\frac{\mathrm{d} \rho}{\mathrm{d} z}\right)^{2}}=\varepsilon \int_{0}^{l} \pi \mathrm{d} z \rho^{2} a_{1}^{\prime}(z) .
\end{aligned}
$$


Apart from the factor $a_{1}{ }^{\prime}(z)$, the integral is just the volume $V$ of the body. In fact, the whole determination of the electric dipole moment resolves itself into the determination of the factor $a_{1}(z)$ in

$$
E=a_{1}(z) \cos \phi
$$

since the other terms in the series do not contribute. If the body is elongated along the axis of symmetry (i.e., if $l \gg p$ ), $a_{1}{ }^{\prime}(z)$ will be a slowly varying function of $z$ and can be removed from the integral and replaced by a mean value (or actually by an estimate of its value). To estimate $a(z)$, we resort to an analogy with reflection from a plane. In the latter case the amplitude of the total field is twice that of the incident field. Thus we choose $a=2 E_{0}$ (phase differences in the incident field at various points on the body can be neglected) to obtain

$$
\boldsymbol{p}=\hat{x} 2 \varepsilon E_{0} V .
$$

The far-zone electric field at a point on the $z$-axis due to the electric dipole is ${ }^{2}$ )

$$
\boldsymbol{E}=-\frac{k^{2}}{4 \pi \varepsilon} \hat{z} x(z \times \boldsymbol{p}) \frac{\mathrm{e}^{i(k r-\omega t)}}{R} .
$$

The form of the magnetic dipole far-zone field is the same as that for the electric dipole if the electric and magnetic fields are interchanged ${ }^{2}$ ). The symmetry of the problem insures that the magnetic dipole is along the $y$-axis, just as the electric dipole is along the $x$-axis. Consequently, the far-zone fields due to the two dipoles have the same orientation and phase. If we again resort to a cylinder-like model for approximation (with the amplitude of the total field at the surface twice that of the incident field), it is obvious from the complete symmetry of occurrence of the electric and magnetic interactions that the two contributions are equal.

Altogether we have in the far zone on the $z$-axis

$$
\boldsymbol{E}=\hat{x} \frac{k^{2}}{\pi} E_{0} V \frac{\mathrm{e}^{i(k R-\omega t)}}{R}
$$

The back-scattering cross-section is given by

$$
\sigma=4 \pi R^{2}\left|\frac{\boldsymbol{E}}{\boldsymbol{E}_{0}}\right|^{2}=\frac{4}{\pi} k^{4} V^{2} .
$$


This, then, is the value of the cross-section to be-expected for an elongated body of revolution*). As the flatness of the scatterer increases, the approximation is expected to get worse; in fact an infinitely flat body (i.e. a disc) has zero volume, but a non-zero cross-section. To anticipate the discussion below, for prolate spheroids the error incurred in the cross.-section varies from zero for extreme elongation to $13 \%$ for the sphere.

Let us now compare this pseudo-derivation in detail with the exact answer for the special case we do know, the spheroid ${ }^{1}$ ). Let us define for convenience the quantity

$$
F=\frac{\pi R}{k^{2} E_{0} V}
$$

$F=1$ yields the magnitude of $\boldsymbol{E}$ given by (2-10). Modifying Rayleigh's notation slightly,

$$
F=\frac{1}{2}\left(\frac{1}{L}+\frac{1}{2-L}\right)=\frac{1}{L(2-L)},
$$

where for a prolate spheroid 1 )

$$
L=\frac{1}{e^{2}}-\frac{1-e^{2}}{2 e^{3}} \ln \frac{1+e}{1-e}
$$

with $e=$ eccentricity; i.e. the semi-axes are $a, a, a / \sqrt{1-e^{2}}$. For an elongated spheroid $(e \rightarrow 1), L \rightarrow 1$ and $F \rightarrow 1$, checking the approximation.

Next, let us inquire into the shape correction by first examining its form for the spheroid. We already know the result for the prolate case; for the oblate spheroid ${ }^{1}$ )

$$
L=\left(\frac{\sqrt{1-e^{2}}}{e^{3}} \sin ^{-1} e-\frac{1-e^{2}}{e^{2}}\right),
$$

where the semi-axes are now. $a, a, a \sqrt{1-e^{2}}$. As these expressions are quite complicated, it is profitable to examine their limiting

*) It should be noted that for the acoustic case the treatment would be equivalent except that instead of the two components (electric and magnetic) there would be only one, and thus the cross-section would be

$$
\sigma=\frac{1}{\pi} k^{4} V^{2}
$$

Appl. sci. Res. B 7 
values. Consider a sphere $(e=0)$ : From (2-14)

$$
\begin{gathered}
\ln \left(\frac{1+e}{1-e}\right)=2\left(e+\frac{1}{3} e^{3}+\ldots\right), \\
L=\frac{1}{e^{2}}\left[1-\left(1-e^{2}\right)\left(1+\frac{1}{3} e^{2}+\ldots\right)\right]= \\
=\frac{1}{e^{2}}\left[1-1+e^{2}-\frac{1}{3} e^{2}+\ldots \ldots\right] \rightarrow \frac{2}{3}, \\
F=\left[\frac{2}{3}\left(2-\frac{2}{3}\right)\right]^{-1}=\left[\frac{2}{3}: \frac{4}{3}\right]^{-1}=\frac{9}{8} .
\end{gathered}
$$

It is easily demonstrated that $F$ is monotonically decreasing as we progress from a sphere to an elongated prolate spheroid. Hence, it ranges from $\frac{9}{8}$ to 1 , being very nearly constant, of the form $1+$ + decaying term.

Examine the disc limit ( $e \rightarrow 1$ for the oblate spheroid). Let

$$
e^{\prime \prime}=\sin x \text {. }
$$

Then

$$
L=\cos x \csc ^{2} x(x \csc x-\cos x)
$$

Let

Then

$$
y=\frac{1}{2} \pi-x
$$

$$
L=\cdot \sin y \sec ^{2} y\left[\left(\frac{1}{2} \pi-y\right) \sec y-\sin y\right] .
$$

Expand near $y=0$ (equivalent to $e \rightarrow 1$ ):

$$
\begin{gathered}
L \approx y\left[\left(\frac{\pi}{2}-y\right)-y\right]=\frac{\pi y}{2}-2 y^{2}=\frac{\pi}{2} y\left(1-\frac{4}{\pi} y\right), \\
F=\frac{1}{L(2-L)} \approx \frac{1}{(\pi / 2) y(1-(4 / \pi) y)(2-(\pi / 2) y)} \approx \\
\approx \frac{1}{\pi y}\left(1+\frac{4}{\pi} y+\frac{\pi}{4} y\right) .
\end{gathered}
$$

For small $y, y \approx \sqrt{1-e^{2}} ;$ if we call the semi-axes $a, a, b$, then $y \approx b / a$.

Combine the information about $F$. In the oblate case, $F$ is again monotonically increasing toward the disc limit. The discussion for the prolate spheroid indicates that we should split off from $F$ a term unity, and that the remaining term should decay as $b / a \rightarrow \infty$. Thus, 
we write

$$
F \approx 1+\frac{1}{\pi y}\left[1+\left(\frac{4}{\pi}+\frac{\pi}{4}-\pi\right) y\right] \approx 1+\frac{1}{\pi y}(1-y) \approx 1+\frac{1}{\pi y} \mathrm{e}^{-y}
$$

We now postulate that for all spheroids (with semi-axes $a, a, b$ ) the shape correction factor is approximately

$$
F=1+\frac{1}{\pi y} \mathrm{e}^{-y}
$$

where $y=b / a$. Numerical comparison indicates that the approximation is valid to within one percent. The Rayleigh cross-section of a spheroid for back-scattering along the axis of symmetry is

$$
\sigma=\frac{4}{\pi} k^{4} V^{2}\left[1+\frac{1}{\pi y} \mathrm{e}^{-y}\right]^{2} .
$$

The cross-section of the spheroid depends on its volume and on a correction factor involving $y=b / a$. Except for very flat oblate spheroids the shape correction factor can be neglected. Where it is not neglected, the shape correction factor is a simple function of $y$, which is a measure of the elongation.

The natural extension of the discussion is to postulate that for all bodies of revolution the Rayleigh cross-section for back-scattering along the axis of symmetry can be expressed as

$$
\sigma=\frac{4}{\pi} k^{4} V^{2}\left[1+\frac{1}{\pi y} \mathrm{e}^{-y}\right]^{2},
$$

where $y$ is a measure of the elongation (characteristic dimension along the axis of symmetry)/(characteristic dimension in the perpendicular direction). For elongated bodies the term in $y$ drops out and there is no ambiguity. For flattened bodies the answer is sensitive to the choice of charcteristic dimensions, but a good approximation should still be attainable. The ambiguity can be eliminated in a number of cases by imposing a restriction on the choice of characteristic dimensions: in the limit of extreme flattening the cross-section must tend to the value for the appropriate disc.

Illustration I: Finite cone. Consider a right circular cone of altitude $h$ and radius of base $r$. As $h \rightarrow 0$, the cross-section of the cone must go into the cross-section of a disc of radius $r$; i.e., we 

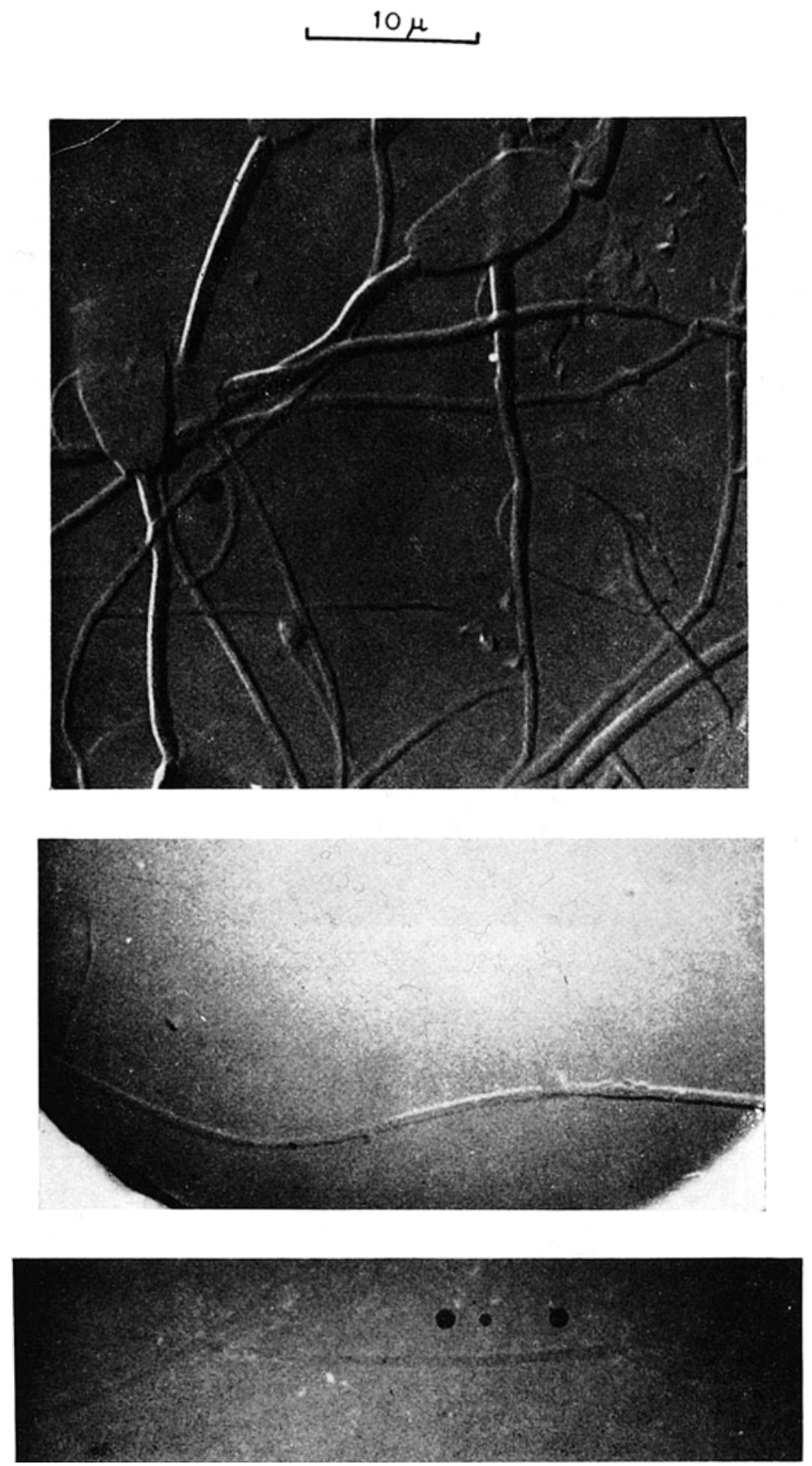

FIG. 17. X ray micrograph of gold shadowed bull sperms. Total magnification appr. $2000 \mathrm{x}$. Exposure conditions as fig. 14 . a. heavily shadowed, b. slightly shadowed, c. X-ray micrograph of untreated bull sperms. Total magnification appr. $2000 \mathrm{x}$. 


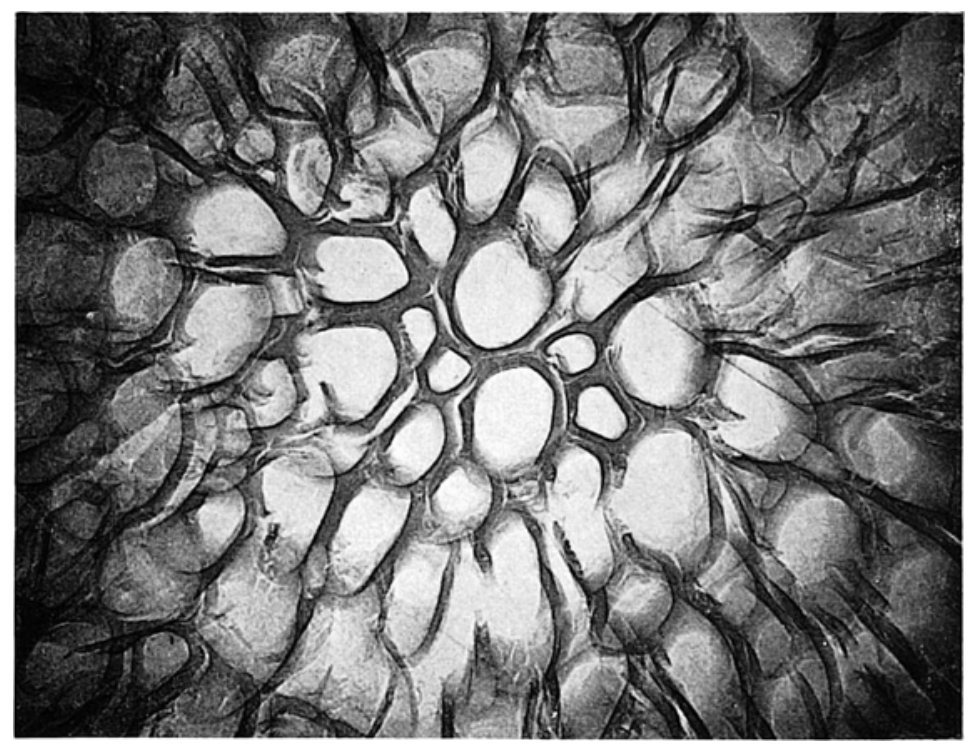

FIG. 18. X-ray micrograph of a transverse section of ashwood, $50 \mu$ thick. Au target ó $\mathrm{kV}$, total magnification appr. $400 \mathrm{x}$. 


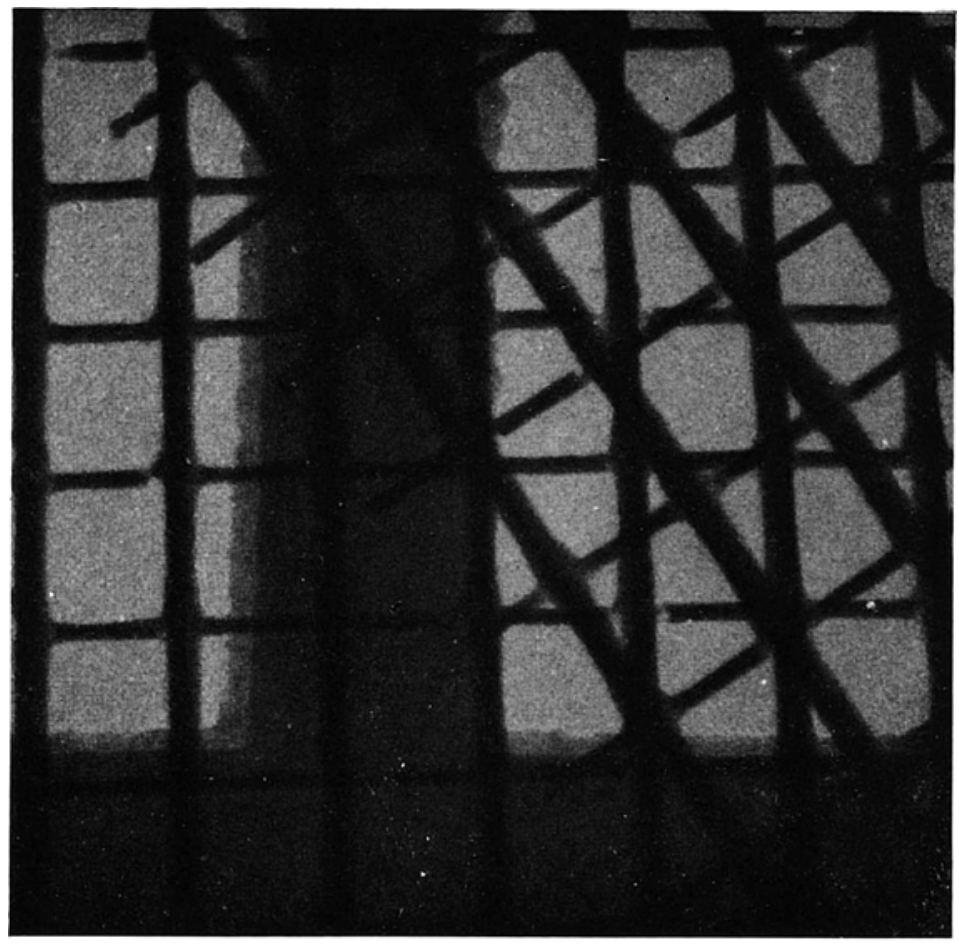

$$
10 \mu
$$

FIG. 16. X-ray micrograph of 1500 mesh silvergrid mounted on 200 mesh copper grid. $\mathrm{Cu}$ target $12 \mathrm{kV}$, total magnification appr. $900 \mathrm{x}$. 
must have

$$
V F=\frac{1}{3} \pi r^{2} h\left(1+\frac{1}{\pi y} \mathrm{e}^{-y}\right) \rightarrow \frac{r^{2} h}{3 y}=\frac{4}{3} r^{3} .
$$

Thus the appropriate ratio of characteristic dimensions to be used in $(2-26)$ is

$$
y=h / 4 r .
$$

Hence, the cone has the same cross-section as a spheroid of equal volume whose semi-axes are $(r, r, h / 4)$.

Illustration II: Lens. Consider à symmetrical convex lens of radius of curvature $R$ (the body of revolution obtained by rotating the shaded area in fig. 2.1 about the $\eta$-axis). In the disc limit $(d$ constant, $c \rightarrow 0$ )

$$
V F \rightarrow V / \pi y=\frac{4}{3} d^{3} .
$$

Hence we take for the lens

$$
y=\frac{3 V}{4 \pi d^{3}}=\frac{3 V}{4 \pi R^{3} \sin ^{3} \theta} .
$$

The volume of the lens is

$$
V=\frac{2}{3} \pi R^{3}(1-\cos \theta)\left(1-\cos \theta+\sin ^{2} \theta\right) .
$$

As $\theta \rightarrow \pi / 2$ (limit of sphere), we reproduce the previous result for the spheroid, as expected.

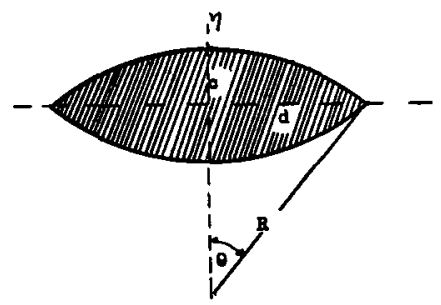

Fig. 2.1 The lens.

Illustration III: Elliptic ogive. Inasmuch as the circular ogive is more elongated than a sphere, the argument from the disc limit cannot be applied to it directly. Instead, we consider the elliptic ogive obtained by rotating the shaded area of fig. 2.2 (a portion of an ellipse) about the $\eta$-axis (which is taken parallel to the 
minor axis). For this body, in the disc limit ( $d$ constant, $c \rightarrow 0$ )

$$
V F \rightarrow V / \pi y=\frac{4}{3} d^{3} \text {. }
$$

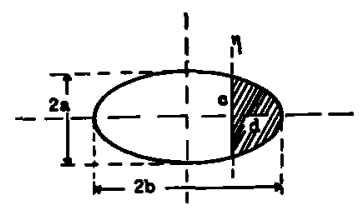

Fig. 2..2 The elliptic ogive.

The equation for the ellipse is

$$
c^{2} / a^{2}+(b-d)^{2} / b^{2}=1
$$

which suggests use of the parameter $\theta$ :

$$
\sin \theta=c / a \text {. }
$$

Then

$$
y=\frac{3 V}{4 \pi d^{3}}=\frac{3 V}{4 \pi b^{3}(1-\cos \theta)^{3}} .
$$

The volume of the elliptic ogive is

$$
V=2 \pi a b^{2}\left(\sin \theta-\theta \cos \theta-\frac{1}{3} \sin ^{3} \theta\right) .
$$

As $\theta \rightarrow \frac{1}{2} \pi$, we reproduce the previous result for the spheroid, as expected.

Special case: Circular ogive. To obtain the cross-section of the circular ogive, we now merely take the special case of the elliptic ogive with $a=b$. From geometry $\theta$ can then be identified with the ogive half-angle. Now

$$
y=\frac{3}{2} \frac{\sin \theta-\theta \cos \theta-\frac{1}{3} \sin ^{3} \theta}{(1-\cos \theta)^{3}} .
$$

Illustration IV: Spindle. Consider the body of revolution obtained by rotating the shaded area of fig. 2.3 (bounded by a parabola and a straight line perpendicular to the axis of the parabola) about the $\eta$-axis. Using the disc limit just as before, we have

$$
y=\frac{3 V}{4 \pi d^{3}},
$$


where the volume is

$$
V=\frac{16}{16} \pi c d^{2}
$$

so that

$$
y=\frac{4}{5} \frac{c}{d} \text {. }
$$

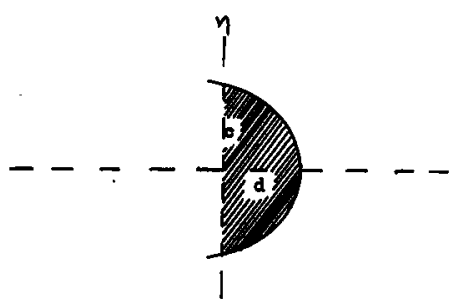

Fig. 2.3. The' spindle.

Illustration V: Finite cylinder. Consider a cylinder of radius $r$ and heigth $h$. From the disc limit

$$
y=\frac{3 V}{4 \pi r^{3}}=\frac{3 h}{4 r} \text {. }
$$

We can go on to obtain by further exploitation of this approach the Rayleigh cross-section of a body of revolution for arbitrary separation between transmitter and receiver and for all aspects and all polarizations. The most direct extension is to replace the body by an equivalent spheroid and take over the spheroid results. The equivalent spheroid is a spheroid with the same volume and the same elongation factor as the body. The simplified expression found for back-scattering along the symmetry axis provides a reasonable way to arrive at an elongation factor for many bodies. The logical ultimate extension in the spirit of this approach is to formulate the Rayleigh scattering of a body of revolution at all aspect combinations and polarizations in terms of the following parameters only: the volume, the elongation factor, and the aspect and polarization angles.

§ 3. The optics region. By the optics region we mean, generally, that region in wavelength, wherein the techniques of geometric and physical optics yield good approximations to the radar cross-section of a body. The extent of the optics region thus depends on the parti- 
cular body being studied. By the geometric optics cross-section we mean $\pi R_{1} R_{2}, R_{1}$ and $R_{2}$ being the principal radii of curvature of the body at the point where a ray is reflected toward the receiver. We use physical optics (Kirchhoff) theory to denote the scattered far field, and the cross-section thus defined, given by the expression

$$
\boldsymbol{H}_{\boldsymbol{s}}=\frac{1}{4 \pi} \int_{\substack{4 \pi \text { uminated } \\ \text { area }}}(\boldsymbol{n} \times \boldsymbol{H}) \times \nabla \frac{\mathrm{e}^{i k \boldsymbol{R}}}{R} \mathrm{~d} s,
$$

where $\boldsymbol{H}=$ twice the tangential component of the incident magnetic field, $R=$ the distance from the integration point to the field point, $\boldsymbol{n}=$ the unit outward normal to the surface at the integration point and in which the far field approximations for $\nabla\left(\mathrm{e}^{i k R / R)}\right.$ are used. That is, with the receiver at a very great distance from the body and if the body is finite, we have

$$
\nabla\left(\frac{\mathrm{e}^{i k R}}{R}\right) \approx \frac{i k}{R^{\prime}}\left(\mathrm{e}^{i k R}\right) \boldsymbol{n}_{0},
$$

where $R=R^{\prime}+n_{0} \cdot r, R^{\prime}=$ the distance from the origin to the field point (receiver), $r=$ the distance from the origin to the integration point on the scatterer ( $\boldsymbol{r}=$ the corresponding vector) and $\boldsymbol{n}_{\mathbf{0}}=$ the unit vector directed from the receiver to the origin.

When the wavelength is small with respect to all of the dimensions of the scatterer, the geometric optics cross-section is an excellent approximation to the exact result. When a body is infinite in extent, then geometric optics can be the exact solution. Examples of such exact solutions are the paraboloid of revolution, when we are considering plane wave illumination along the axis of symmetry, and the wedge for particular wedge angles and for particular angles of -incidence and polarization.

Let us now consider a body which has one radius of curvature which is small with respect to the wavelength. In three dimensions we can consider the infinite cone and in two dimensions we can consider the wedge. By purely dimensional analysis we find that the tip far field behaves like $1 / k$ and the edge in two dimensions behaves like $(1 / k)$. We find that physical optics not only predicts these types of $k$-dependence, but also (for large and small cone angles) that it predicts the leading term of a rapidly convergent 
expansion in the angle parameter as long as the transmitter or receiver is on the axis of symmetry.

Kirchhoff theory will predict poor results for problems in which the major contribution to the cross-section comes from an edge. For example, consider the case in which the transmitter and receiver are located at a point along the face of a wedge, but far from the edge, with Poynting's vector $\boldsymbol{P}$ parallel to the face of the wedge and normal to the edge (see fig. 3.1). For the $\boldsymbol{E}$-vector perpendicular to the surface the exact result is

$$
\sigma_{\perp}=2 \pi|f(\phi)|^{2}=\frac{\pi \lambda}{2 \phi_{0}^{2}} \operatorname{tàn}^{2} \frac{\pi^{2}}{2 \phi_{0}},
$$

where the cross-section, in two dimensions, is given by

$$
\sigma=\lim _{r=\infty} 2 \pi r\left|\frac{E_{s}}{E_{i}}\right|^{2},
$$

while the Kirchhoff answer is zero.

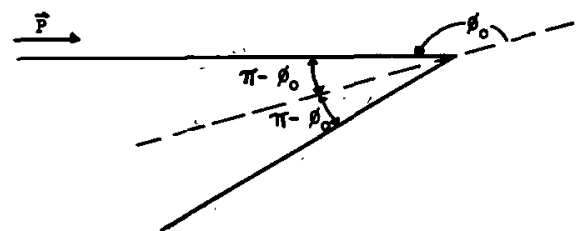

Fig. 3.1. The wedge for incidence along one face of the wedge and normal \{. to the edge.

This leads one to the realization of why Kirchhoff theory would give poor results for a finite thin cone. The major contribution to the cross-section in the non-specular directions for small wavelengths comes from the rear circular edge. The field, locally, would be like that for a wedge. Thus, we need to use an improvement to Kirchhoff theory to obtain good answers for the cone. We will show this improvement and also how we obtain approximate results for thin cones in the resonance region. Thus we will show how to obtain, approximately, a complete cone cross-section curve.

Kirchhoff theory gives excellent first order approximations for bodies with dimensions large with respect to the wavelength, and these results are too well-known to warrant their discussion here. In the region to which we must give the vague characterization as 
lying somewhere between the resonance region and the optics region there has been a rapid and fruitful development of trew ideas during the past ten years.

We begin with the remarkable paper of V. A. F ock ${ }^{3}$ ) in which he presented a method which we will describe as a local order analysis of the field near the shadow boundary. He succeeds in giving the fields on the diffracting surface near the shadow boundary in terms of one or the other of two "universal" functions according as the incident polarization direction lies parallel or perpendicular to the shadow curve. Strictly, these are solutions of the two-dimensional (scalar) problems and depend on the radius of curvature at the shadow boundary and the wavelength of the radiation. These functions are of the form

$$
\begin{aligned}
& g(\zeta)=\frac{1}{\dot{V} \pi} \int_{\Gamma} \frac{\mathrm{e}^{i \zeta t}}{w^{\prime}(t)} \mathrm{d} t, \\
& f(\zeta)=\frac{1}{\sqrt{ } \pi} \int_{\Gamma} \frac{\mathrm{e}^{i \zeta t}}{w(t)} \mathrm{d} t
\end{aligned}
$$

where

$$
w(t)=\frac{1}{\sqrt{ } \pi} \int_{r^{\prime}} \mathrm{e}^{z t-t z^{3}} \mathrm{~d} z
$$

with the contours shown in fig. 3.2. The arguments used are certain
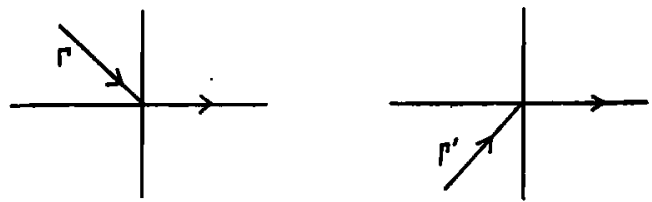

Fig. 3.2. Contours.
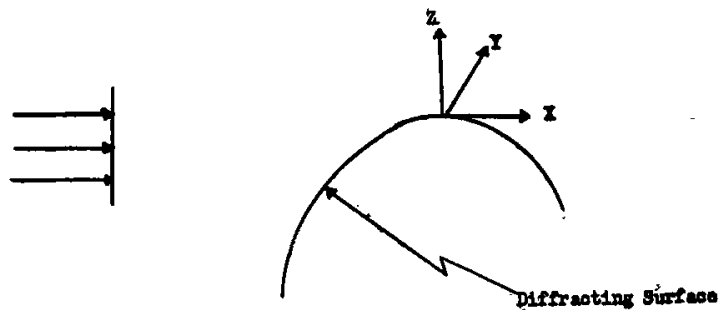

Fig. 3.3. Geometry. 
reduced distances measured from the geometrical shadow boundary; i.e., near the origin as indicated in fig. 3.3 we have

$$
\zeta=\left(\frac{k R}{2}\right)^{\ddagger} \frac{X}{R}
$$

where $R$ is the radius of curvature and $k=2 \pi / \lambda$.

These same functions appear in the approximate solutions of specific problems. There are two which we particularly wish to note. The fields induced on a parabolic cylinder ${ }^{4}$ ) and on a circular cylinder 5) are given, in a sense, by these same functions. These are not remarkably similar surfaces.

In these examples for the solution continued into the shadow we need to modify the arguments of the universal functions as follows. The motivation for this stems from the "generalized ray optics" of Keller $\left.{ }^{6}\right)$. In place of (3-4) we write

$$
\zeta=\int_{0}^{S}\left[\frac{k R(s)}{2}\right]^{\xi} \frac{\mathrm{d} s}{R(s)},
$$

where $S$ is the path length measured along the surface of the obstacle from the shadow boundary into the shadow, $\mathrm{d} s$ is the element of path length and $R(s)$ is the radius of curvature at the position $s$.

Franz and Deppermann ?), however, have given the connection between the two in the concept of "creeping waves". We can meaningfully speak of the continuation of the penumbra solution into the shadow of the parabolic cylinder, but in the case of the circular cylinder we find that we are wrapping our solution around the cylinder if we allow the argument of the universal functions to continue increasing. This latter concept is made meaningful if we understand the field in the shadow as arising from waves "launched" at the shadow boundary and "creeping" around the rear and eventually back to the front, etc. The physical interpretation has been justified by Friedlander ${ }^{8}$ ) while the underlying mathematical structure has been illuminated by $\mathrm{Wu}^{9}$ ) with his concept of a universal covering space.

In the following is given an account of the general procedure. Let a convex closed surface $S, f(x, y, z)=0$ be illuminated by a plane wave incident in the direction of the $x$-axis. The geometrical shadow is then given by the two equations $f(x, y, z)=0, \partial f / \partial x=0$. Let the 
origin be located at a point on the shadow boundary with the $z$-axis the outward normal to $S$ and the $y$-axis chosen to form a righthanded system. Using the geometric assumption that the surface can be approximated by a paraboloid at any point, i.e.,

$$
z+\frac{1}{2}\left(a x^{2}+2 b x y+c y^{2}\right)=0,
$$

so that $\partial f / \partial x=a x+b y$, and the physical assumption that the variation of this field in the $z$ direction is much larger than that in either the $x$ - or $y$ - direction for sufficiently small $\lambda$, Fock obtains an approximation to Maxwell's equations which lead to the solutions

$$
H_{y}=H_{y}^{0} G(\zeta), \quad H_{x}=(2 a \mid k)^{\frac{1}{3}} i H_{z}^{0} \mathrm{e}^{i k x} F(\zeta), \quad H_{z}=0
$$

on the surface. The incident field is given by

$$
\boldsymbol{H}_{0}=\left(0, H_{y^{0}}, H_{z}{ }^{0}\right),
$$

while the functions $G$ and $F$ have the asymptotic bahaviour

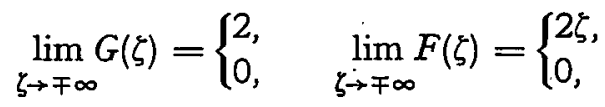

where $\zeta$ is a reduced distance from the shadow boundary given by

$$
\zeta=\left(\frac{k}{2 a}\right)^{\xi}(a x+b y) .
$$

In fig. 3.4 we compare the result using the Fock-Franz method with the sum of the harmonic series for a circular cylinder (e.g., Bailin's work in ${ }^{10}$ )) with $k a=12$. In fig. 3.5. we compare the method with the experimental measurements of Wetzel and Brick ${ }^{11}$ ) on an elliptic cylinder of $k a=12$ and $k b=7.5$.

In the case of the three-dimensional problem of scattering by finite obstacles we have an additional complication which appears in both the scalar and vector problems: Since there is a caustic at the rear of the obstacle, we must take account of the fact that the energy converges on the caustic and, in fact, the "creeping waves" lose their identity in this region.

This behaviour is apparent from the work of Fock ${ }^{12}$ ), Franz ${ }^{5}$ ) and, more recently, Belkina and Weinstein ${ }^{13}$ ) and N.. Logan 14) who have given a thorough treatment of thiş approach for the sphere. However, Fock theory can be used to determine a partial creeping 
wave type field, and if we can find another way to handle the partial field due to the small radii of curvature, we can again obtain good far-field approximations formoderate values of $k a$. The value of Fock theory is twofold: (1) When the wavelength is very small in respect to the characteristic dimensions of the body, it yields an approximation to the true field in the shadow region where the Kirchhoff result would predict a zero field, and (2) it is a procedure which is easily applied to sphere and cylinder problems for moderate values of $k a(k a>5)$. One finds upon applying this process to spheroids that the values of $k a$ required in order to obtain good results may be very large.

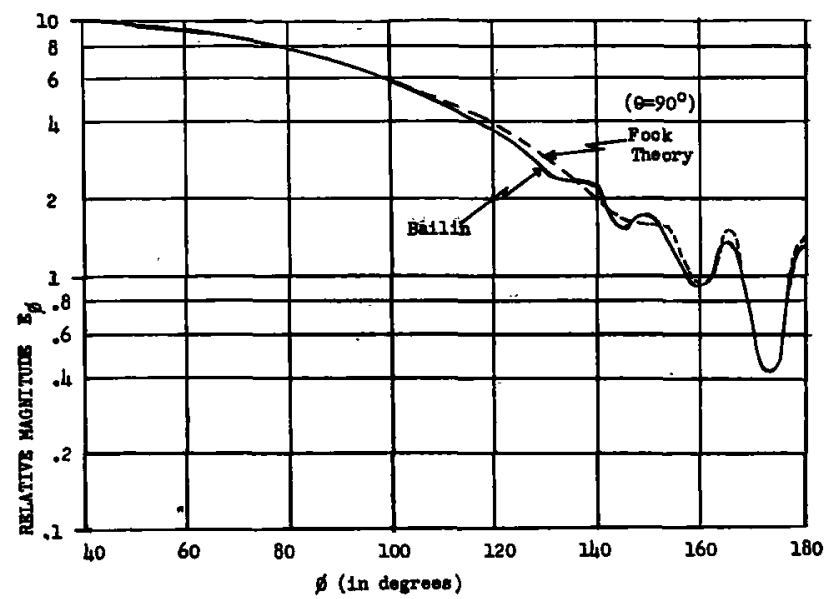

Fig. 3.4. Comparison of amplitudes from exact series and Fock's current distribution for a circular cylindér with $k a=12$.

In the three-dimensional problems we see that the solution for the sphere with the interpretation of creeping waves and behaviour of the caustic serves as a prototype from which we infer the solution for other shapes provided the characteristic parameters are sufficiently large with respect to the wavelength. For example, a symmetrically illuminated spheroid of large enough dimensions should be an easy generalization.

Suppose we consider a prolate spheroid in somewhat more detail. Let the semi-major and semi-minor axes be denoted by $a$ and $b$ respectively. The condition that we require to be met for the application of the Fock-Franz theory is that $k R_{\min }$ be large where 
$k=2 \pi / \lambda$ and $R_{\min }$ is the minimum radius of curvature, $R_{\min }=b^{2} / a$. As an example of this limitation we note that for a prolate sphereid of $a / b=10$ the requirement $k R_{\min } \sim 5$ would imply $k a>500$. This was pointed out by Belkina and Weinstein ${ }^{13}$ ).

If we let $k R_{\min }$ decrease while we keep $k a, k b$ large, we approach a body which is "large" but which has "sharp" ends. We illuminate this object along the symmetry axis and consider a limited applica-

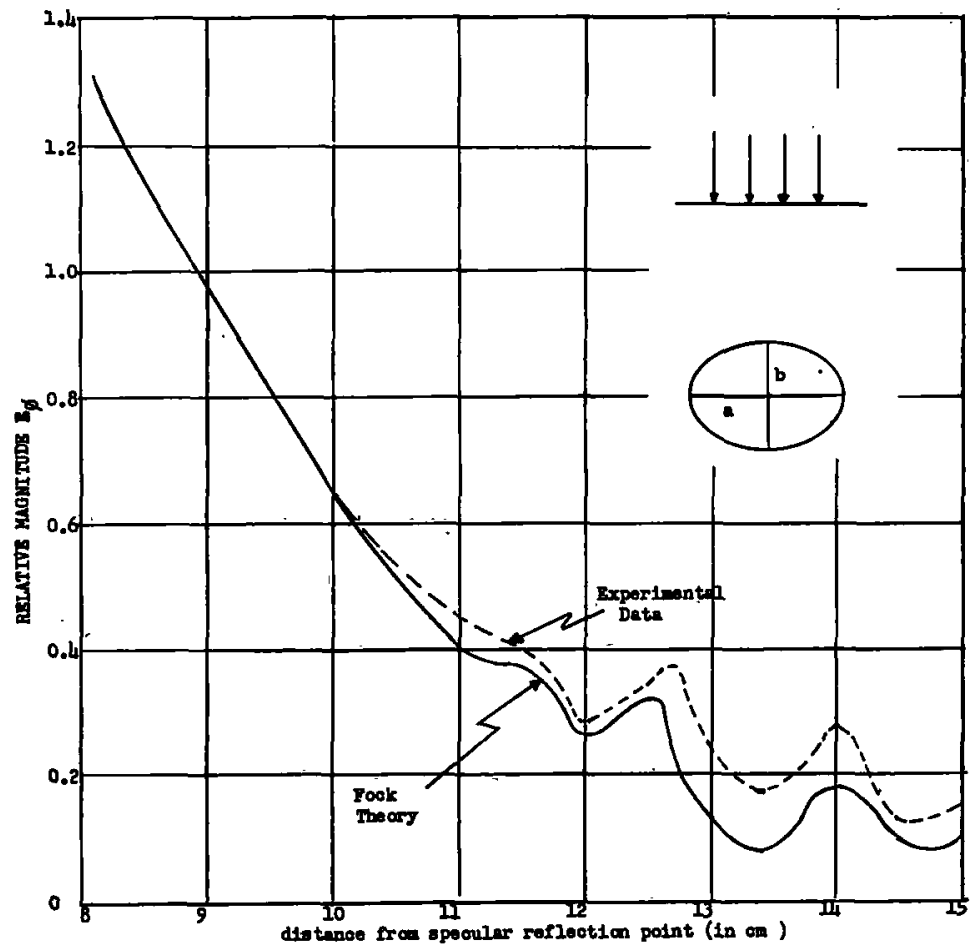

Fig. 3.5. Comparison of amplitudes from experimental data and Fock's current distribution for an elliptic cylinder of eccentricity 0.780 with $k a=12$ and $k b=7.5$.

tion of our "creeping wave" theory. Certainly for $k R_{\min }<1$ the forward tip will scatter more like an infinite cone than like a sphere of radius $b$; hence, our theory is not applicable. In the penumbra region all requirements are met and we feel justified in making a creeping wave analysis. Granted this, we have launched a wave which is creeping toward this effective discontinuity., the rear tip. Here we must again have recourse to another description and consider the 
wave to be reflected from the rear tip and again launched along the surface.

An example of this would be the thin cone radiation problem when the source is far from the! tip ( $a=$ distance from tip to source). The Green's function for this case is approximated by that for a cylinder and thus Fock theory should give excellent results. If the infinitesimal slot is along a generatrix of the cone, the Fock answer should be excellent for all $k a$. If one can obtain an tip answer to add to the Fock result, then one could handle all kinds of slots on cones. We postpone our discussion of the ogive, finite cone and the spheroid approximations (for moderate values of $k a$ ) until we reach the discussion of the resonance region in $\S 4$.

In addition to the Fock theory, small wavelength approximations can be improved by making use of known results. Just as Art$\operatorname{mann}{ }^{15}$ ), in his solution for the thick half plane, replaced the cylindrical edge by a polygon, we can obtain an approximation for the thin finite cone by replacing the cone by a regular prism. The base, locally, will be a wedge, and to calculate the field scattered by the cone base we will add up the fields scattered by all the wedge-like segments into which the cone base has been decomposed. We shall consider the cone in some detail; hence, it might be valuable to first present the physical optics approximation.

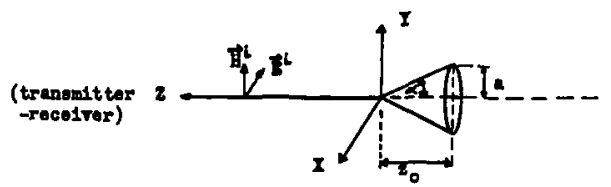

Fig. 3.6. Cone geometry.

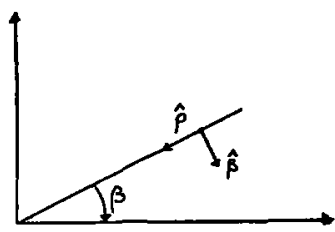

Fig. 3.7. Polar variables.

The problem we shall consider is that of determining the radar cross-section of a thin finite cone when both transmitter and receiver are situated on the axis of symmetry of the cone in the far 
zone. We will treat the case where the wavelength of the incident radiation is much smaller than the altitude $z_{0}$ and the base radius $a$ of the cone. The geometry of the problem is as shown in fig. 3.6. We shall also utilize polar variables in the $x-y$ plane as shown in fig. 3.7.

The following definitions of the radar cross-section (of perfect conductors) will be used:

$$
\sigma=\lim _{r \rightarrow \infty} 4 \pi r^{2}\left|\frac{\boldsymbol{E}^{s}}{\boldsymbol{E}^{i}}\right|^{2}=\lim _{r \rightarrow \infty} 4 \pi r^{2}\left|\frac{\boldsymbol{H}^{s}}{\boldsymbol{H}^{i}}\right|^{2} .
$$

The Kirchhoff (physical optics) expression for the scattered magnetic field is ${ }^{16}$ )

$$
\boldsymbol{H}^{s}=\frac{i k}{2 \pi} \frac{e^{-i k r}}{r}\left[\left(\boldsymbol{n}_{0} \cdot \boldsymbol{p}\right) \boldsymbol{f}-\left(\boldsymbol{n}_{0} \cdot \boldsymbol{f}\right) \boldsymbol{p}\right]
$$

where

$$
\begin{aligned}
& \boldsymbol{f}=\int_{S} \boldsymbol{n} \mathrm{e}^{-i k r \cdot\left(\boldsymbol{n}_{0}+\boldsymbol{k}\right)} \mathrm{d} s \\
& S=\text { illuminated area of scatterer } \\
& \boldsymbol{n}=\text { unit } \text { 'outward normal to } S \\
& \boldsymbol{r}=\text { position vector of point on } S \\
& \boldsymbol{p}=\text { direction of incident magnetic field } \\
& \boldsymbol{n}_{0}=\text { receiver to origin direction } \\
& \boldsymbol{k}=\text { transmitter to origin direction. }
\end{aligned}
$$

Note. we assume $\left|H^{i}\right|=\left|E^{i}\right|=1$. In this case, the following relations hold.

$$
\begin{aligned}
& \boldsymbol{n}=\sin \alpha \hat{\imath}_{z}+\cos \alpha\left(\hat{\imath}_{x} \cos \beta+\hat{\imath}_{y} \sin \beta\right), \boldsymbol{r}=x \hat{\imath}_{x}+y \hat{\imath}_{y}+z \hat{\imath}_{z}, \\
& \boldsymbol{p}=\hat{\imath}_{y}, \quad \boldsymbol{n}_{0}=-\hat{\imath}_{z}, \quad \boldsymbol{k}=-\hat{\imath}_{z}, \quad \mathrm{~d} s=\frac{z \tan \alpha}{\cos \alpha} \mathrm{d} z \mathrm{~d} \beta .
\end{aligned}
$$

Hence

$$
\boldsymbol{n}_{0} \cdot \boldsymbol{p}=-\hat{\imath}_{z} \cdot \hat{\imath}_{y}=0
$$

and

$$
\boldsymbol{n}_{0} \cdot \boldsymbol{f}=\int_{S} \boldsymbol{n}_{0} \cdot \boldsymbol{n} \mathrm{e}^{-i k r \cdot\left(\boldsymbol{n}_{0}+\boldsymbol{k}\right)} \mathrm{d} s,
$$

which becomes

$$
=-\tan ^{2} \alpha \int_{0}^{2 \pi} \int_{0}^{-z_{0}} z \mathrm{e}^{2 i k z} \mathrm{~d} z \mathrm{~d} \beta
$$


The integration with respect to $\beta$ yields

$$
\boldsymbol{n}_{0} \cdot \boldsymbol{f}=-2 \pi \tan ^{2} \alpha \int_{0}^{-z_{0}} z \mathrm{e}^{2 i k z} \mathrm{~d} z .
$$

This integration can also be performed yielding

$$
\begin{gathered}
\boldsymbol{n}_{0} \cdot \boldsymbol{f}=-\left.2 \pi \tan ^{2} \alpha\left(\frac{-\mathrm{e}^{2 i k z}}{(2 i k)^{2}}+\frac{z \mathrm{e}^{2 i k z}}{2 i k}\right)\right|_{0} ^{-z_{0}}, \\
\boldsymbol{n}_{0} \cdot \boldsymbol{f}=-2 \pi \tan ^{2} \alpha\left(-\frac{\mathrm{e}^{-2 i k z_{0}}}{4 k^{2}}-\frac{z_{0} \mathrm{e}^{-i k z_{0}}}{2 i k}-\frac{1}{4 k^{2}}\right) .
\end{gathered}
$$

Hence

$$
\boldsymbol{H}^{s}=\frac{i e^{-i k r}}{r} \tan ^{2} \alpha k \hat{\imath}_{y}\left(\frac{\mathrm{e}^{-2 i k_{0}}}{4 k^{2}}-\frac{z_{0} \mathrm{e}^{-2 i k z_{0}}}{2 i k}-\frac{1}{4 k^{2}}\right),
$$

which can be written

$$
\boldsymbol{H}^{8}=\frac{i \mathrm{e}^{-i k\left(r+2 z_{0}\right)}}{2 k r} \tan ^{2} \alpha \hat{\imath}_{y}^{\hat{r}}\left(\frac{1}{2}+i k z_{0}-\frac{e^{2 i k z_{0}}}{2}\right) .
$$

Since $\left|k z_{0}\right|>1$ (small wavelength approximation)

$$
\boldsymbol{H}^{s} \approx \frac{i \mathrm{e}^{-i k\left(r+2 z_{0}\right)}}{2 k r} \tan ^{2} \alpha \hat{\imath}_{y}\left(i k z_{0}\right)=-\frac{\mathrm{e}^{-i k\left(r+2 z_{0}\right)}}{2 r} z_{0} \tan ^{2} \alpha \hat{\imath}_{y} .
$$

Now using the definition of radar cross-section we have

or

$$
\sigma=\lim _{r \rightarrow \infty} 4 \pi r^{2}\left|\frac{\boldsymbol{H}^{s}}{\boldsymbol{H}^{i}}\right|^{2}=\pi z_{0}^{2} \tan ^{4} \alpha
$$

$$
\sigma=\pi a^{2} \tan ^{2} \alpha .
$$

We will now approximate the cone by a prism and determine the field scattered by the wedge segments that constitute the base.

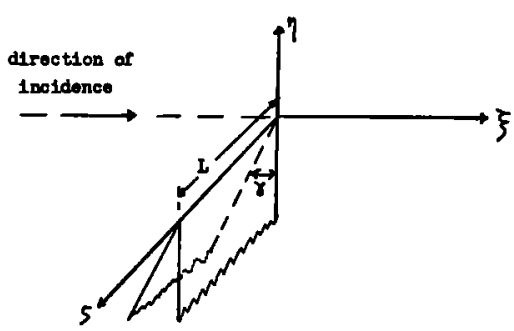

Fig. 3.8. Wedge geometry. 
To illustrate the technique we will first use the physical optics approximation for the field scattered by the wedges. We use the Kirchhoff expressions for the scattered field previously presented where in this case (incidence perpendicular to the back face of the wedge, see fig. 3.8 ) we have

$$
\begin{gathered}
\boldsymbol{n}=\sin \gamma \hat{\imath}_{\eta}-\cos \gamma \hat{\imath}_{\xi}, \boldsymbol{r}=\xi \hat{\imath}_{\xi}+\eta \hat{\imath}_{\eta}+\zeta \hat{\imath}_{\xi}, \\
\boldsymbol{p}=\boldsymbol{p}\left(\text { no } \xi \text { component i.e., } \boldsymbol{p} \cdot \hat{\imath}_{\xi}=0\right), \boldsymbol{n}_{0}=\hat{\imath}_{\xi}, \boldsymbol{k}=\hat{\imath}_{\xi}, \\
\mathrm{d} s=\frac{\mathrm{d} \xi \mathrm{d} \zeta}{\sin \gamma} .
\end{gathered}
$$

Hence $\boldsymbol{n}_{\mathbf{0}} \cdot \boldsymbol{p}=\hat{\imath} \cdot \boldsymbol{p}=0$, and

$$
\boldsymbol{n}_{0} \cdot \boldsymbol{f}=-\frac{\cos \gamma}{\sin \gamma} \int_{0}^{L} \int_{0}^{-\infty} \mathrm{e}^{-2 i k \xi} \mathrm{d} \xi \mathrm{d} \zeta .
$$

Integrating with respect to $\zeta$ and letting $\xi \rightarrow-\xi$, we obtain

$$
\boldsymbol{n}_{0} \cdot \boldsymbol{f}=\frac{L}{\tan \gamma} \int_{0}^{\infty} \mathrm{e}^{2 i k \xi} \mathrm{d} \xi=\left.\frac{L}{\tan \gamma} \frac{\mathrm{e}^{2 i k \xi}}{2 i k}\right|_{0} ^{\infty} .
$$

Associating the edge contribution with the value at the lower limit (just as in the case of the infinite cone we obtain the "tip" contribution) we find

$$
\boldsymbol{n}_{0} \cdot \boldsymbol{f}=-\frac{L}{2 i k \tan \gamma}
$$

Hence

$$
\boldsymbol{H}^{s}=\frac{L}{4 \pi \tan \gamma} \frac{\mathrm{e}^{-i k r}}{r} \boldsymbol{p} .
$$

Now letting $L=a \mathrm{~d} \beta$, where $a=$ radius of base, and integrating around the base ( $\boldsymbol{p}=$ constant vector $)$, we have

$$
\begin{gathered}
\boldsymbol{H}^{s}=\int_{0}^{2 \pi} \frac{\boldsymbol{p} \mathrm{e}^{-i k r}}{4 \pi r \tan \gamma} a \mathrm{~d} \beta=\frac{a p \mathrm{e}^{-i k r}}{2 r \tan \gamma}, \\
\sigma=4 \pi r^{2}\left|\frac{\boldsymbol{H}^{s}}{\boldsymbol{H}^{i}}\right|^{2}=4 \pi r^{2}\left|\frac{a \mathrm{e}^{-i k r}}{2 r \tan \gamma} \boldsymbol{p}\right|^{2}=\frac{a^{2}}{\tan ^{2} \gamma} .
\end{gathered}
$$

Appl. sci. Res. B 7 
But $\gamma=\frac{1}{2} \pi-\alpha$ (see fig. 3.9), where $\alpha$ is half the cone angle; thus, $\tan \alpha=\tan \left(\frac{1}{2} \pi-\gamma\right)=\cot \gamma$ and finally

$$
\sigma=\pi a^{2} \tan ^{2} \alpha,
$$

which is precisely the nose-on result obtained for the cone directly by physical optics.

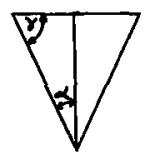

Fig. 3.9. Definitions of $\gamma$ and $\alpha$.

Now we are in a position to employ this technique to obtain a new result for the finite cone. We make use ${ }^{17}$ ) of the following expression for the electric field scattered by an infinite wedge:

$$
\begin{aligned}
& \boldsymbol{E}^{\varepsilon}=\frac{\sqrt{2 \pi / k r}}{2(2 \pi-\gamma)} \cdot \\
& \mathrm{e}^{i k r+i i \pi} \sin \left(\frac{\pi^{2}}{2 \pi-\gamma}\right)\left[\frac{E(a) \hat{\rho}-\mathrm{E}(b) \hat{\beta}}{A}-\frac{E(a) \hat{\rho}+E(b) \hat{\beta}}{B}\right],
\end{aligned}
$$

where incidence is in a direction perpendicular to the edge of the wedge and

$E(a)=E_{\perp}^{i}=$ component of the incident field perpendicular to the edge of the wedge,

$E(b)=E_{\|}^{i}=$ component of the incident field parallel to the edge of the wedge,

$$
\begin{aligned}
& A=\cos \left(\frac{2 \pi \theta}{2 \pi-\gamma}\right)+\cos \left(\frac{\pi^{2}}{2 \pi-\gamma}\right), \\
& B=1-\cos \left(\frac{\pi^{2}}{2 \pi-\gamma}\right),
\end{aligned}
$$

$\theta=$ angle of incidence measured from wedge angle bisector and $\hat{\rho}$ and $\hat{\beta}$ are unit vectors perpendicular and parallel, respectively, to the edge of the wedge.

This expression is valid for an infinite wedge. In order to obtain 
an expression for a wedge of finite edge length, we again look at the current distribution integrals. Noting first that we now have an $\mathrm{e}^{+i k r}$ phase term, we know that the integral over the edge length will be in the two-dimensional case

$$
\int_{-\infty}^{\infty} \mathrm{e}^{i k \sqrt{r^{2}+\zeta^{2}}} \mathrm{~d} \zeta \approx \sqrt{r \lambda} \mathrm{e}^{i(k r+\dot{\xi} \pi)}
$$

and in the three-dimensional case

$$
\int_{0}^{L} \mathrm{e}^{i k \sqrt{r^{2}+\zeta^{2}}} \mathrm{~d} \zeta \approx L \mathrm{e}^{i k r} .
$$

This is the:only difference between the two- and three-dimensional problems so that the three-dimensional fields can be obtained from the two-dimensional fields by multiplying by

$$
\frac{L \mathrm{e}^{-\frac{1}{2} \pi i}}{\sqrt{r \lambda}} \text {. }
$$

Thus we obtain, for linear polarization, the following expression for the scattered field for a wedge of length $L$ :

$$
\begin{aligned}
\boldsymbol{E}^{s} & =\frac{L \mathrm{e}^{i k r}}{2 r(2 \pi-\gamma)} . \\
& . \sin \left(\frac{\pi^{2}}{2 \pi-\gamma}\right)\left[\frac{E(a) \hat{\rho}-E(b) \hat{\beta}}{A}-\frac{E(a) \hat{\rho}+E(b) \hat{\beta}}{B}\right] .
\end{aligned}
$$

Again we are really considering the base of a cone and hence

$$
\hat{\rho}=-\hat{\imath}_{x} \cos \beta-\hat{\imath}_{y} \sin \beta, \hat{\beta}=+i_{x} \sin \beta-\hat{\imath}_{y} \cos \beta .
$$

$\boldsymbol{E}^{\boldsymbol{i}}$, we recall, is equal to $-\hat{\imath}_{x}$, but $\hat{\imath}_{x}=-\hat{\rho} \cos \beta+\hat{\beta} \sin \beta$. Hence

$$
E_{\perp}^{i}=\cos \beta, \quad E_{\|}^{i}=-\sin \beta .
$$

Thus, using these relations for $E_{\perp}{ }^{i}, E_{\|} \|^{i}, \hat{\rho}$ and $\hat{\beta}$,

$$
E_{\perp} i \hat{\rho}-E_{\mid 1} i \hat{\beta}=\hat{\rho} \cos \beta+\hat{\beta} \sin \beta=-\hat{\imath}_{x} \cos 2 \beta-\hat{\imath}_{y} \sin 2 \beta
$$

in rectangular unit vectors and similarly

$$
E_{\perp}^{i} \hat{\rho}+E_{\|}^{i \hat{\beta}}=\hat{\rho} \cos \beta-\hat{\beta} \sin \beta=-\hat{\imath}_{x} .
$$


Substituting in $\boldsymbol{E}^{s}$ we obtain

$$
\begin{aligned}
& \boldsymbol{E}^{\delta}=\frac{L \mathrm{e}^{i k r}}{2 \gamma(2 \pi-\gamma)} \sin \left(\frac{\pi^{2}}{2 \pi-\gamma}\right) . \\
& {\left[-\frac{\hat{\imath}_{x} \cos 2 \beta+\hat{\imath}_{y} \sin 2 \beta}{\cos \left(\frac{2 \pi \theta}{2 \pi-\gamma}\right)+\cos \left(\frac{\pi^{2}}{2 \pi-\gamma}\right)}+\frac{\hat{\imath}_{x}}{1-\cos \left(\frac{\pi^{2}}{2 \pi-\gamma}\right)}\right] .}
\end{aligned}
$$

As before, we set $L=a \mathrm{~d} \beta$ and integrate over $\beta$ from 0 to $2 \pi$, obtaining for the scattered field from the cone

$$
\boldsymbol{E}_{\text {cone }}=\frac{\pi a \mathrm{e}^{i k r}}{r(2 \pi-\gamma)} \sin \left(\frac{\pi^{2}}{2 \pi-\gamma}\right) \frac{\hat{\imath}_{x}}{1-\cos \left(\frac{\pi^{2}}{2 \pi-\gamma}\right)} .
$$

Now, using the definition

$$
\sigma=\lim _{r \rightarrow \infty} 4 \pi r^{2}\left|\frac{\boldsymbol{E}^{8}}{\boldsymbol{E}^{i}}\right|^{2},
$$

we obtain

$$
\sigma=\frac{4 \pi^{3} a^{2}}{(2 \pi-\gamma)^{2}} \frac{\sin ^{2}\left(\frac{\pi^{2}}{2 \pi-\gamma}\right)}{\left[1-\cos \left(\frac{\pi^{2}}{2 \pi-\gamma}\right)\right]^{2}} .
$$

Using the familiar half-angle formulae we can simplify $\sigma$ as follows:

$$
\frac{\sin ^{2}\left(\frac{\pi^{2}}{2 \pi-\gamma}\right)}{\left[1-\cos \left(\frac{\pi^{2}}{2 \pi-\gamma}\right)\right]^{2}}=\cot ^{2}\left[\frac{\pi^{2}}{2(2 \pi-\gamma)}\right] \text {. }
$$

Hence

$$
\sigma=\frac{4 \pi^{3} a^{2}}{(2 \pi-\gamma)^{2}} \cot ^{2}\left[\frac{\pi^{2}}{2(2 \pi-\gamma)}\right] .
$$

In terms of the cone angle $\alpha$ we have, since $\gamma=\frac{1}{2} \pi-\alpha$,

$$
\sigma=\frac{\pi^{3} a^{2}}{\left(\frac{3}{4} \pi+\frac{1}{2} \alpha\right)^{2}} \cot ^{2}\left(\frac{\pi^{2}}{3 \pi+2 \alpha}\right) .
$$


This result is compared with the physical optics result in fig. 3.10. Our method can be applied to any body with a ring singularity.

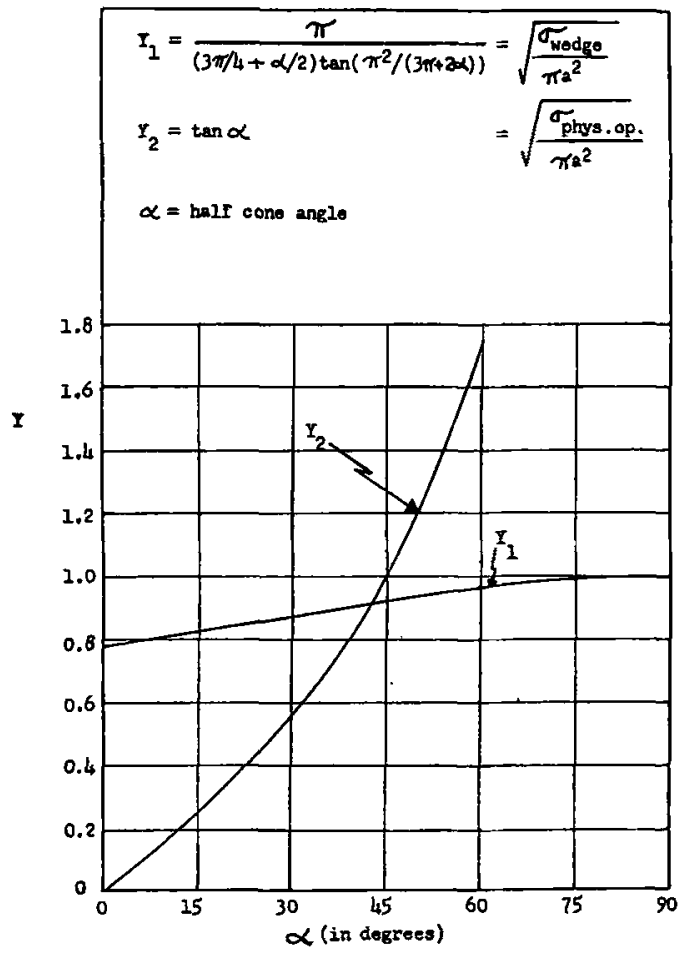

Fig. 3.10. Nose-on finite cross-sections as computed by physical optics and circular wedge approximations.
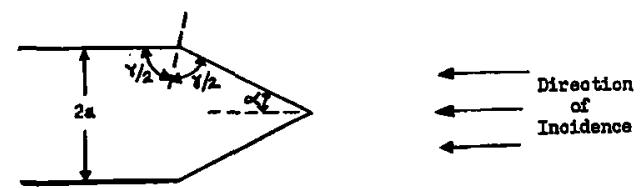

Fig. 3.11. Cone-cylinder combination.

Consider, for example, a cone-cylinder conbination viewed nose-on (see fig. 3.11). The expression derived for the cross-section

$$
\frac{\sigma}{\pi a^{2}}=\frac{\pi^{2}}{\left(\pi-\frac{\gamma}{2}\right)^{2}} \cot ^{2}\left(\frac{\pi^{2}}{4 \pi-2 \gamma}\right)
$$


still applies. In terms of the half cone angle $\alpha$ we have, since $\alpha=\pi-\gamma$,

$$
\frac{\sigma}{\pi a^{2}}=\frac{\pi^{2}}{\left(\frac{\pi}{2}+\frac{\alpha}{2}\right)^{2}} \cot ^{2}\left[\frac{\pi^{2}}{2(\pi+\alpha)}\right] .
$$

Similarly, for the contribution from any ring singularity (3-43) holds, where $\gamma$ is the included wedge angle (fig. 3.12). Equations (3-42), (3-43) and (3-44) are plotted in fig. 3.13.
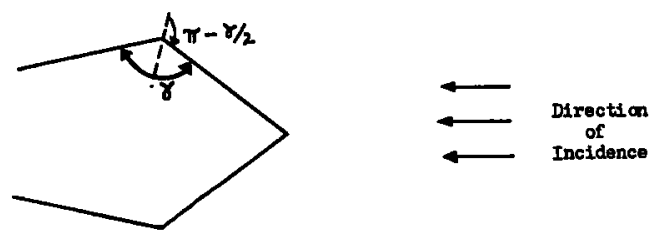

Fig. 3.12. Definition of wedge angle.

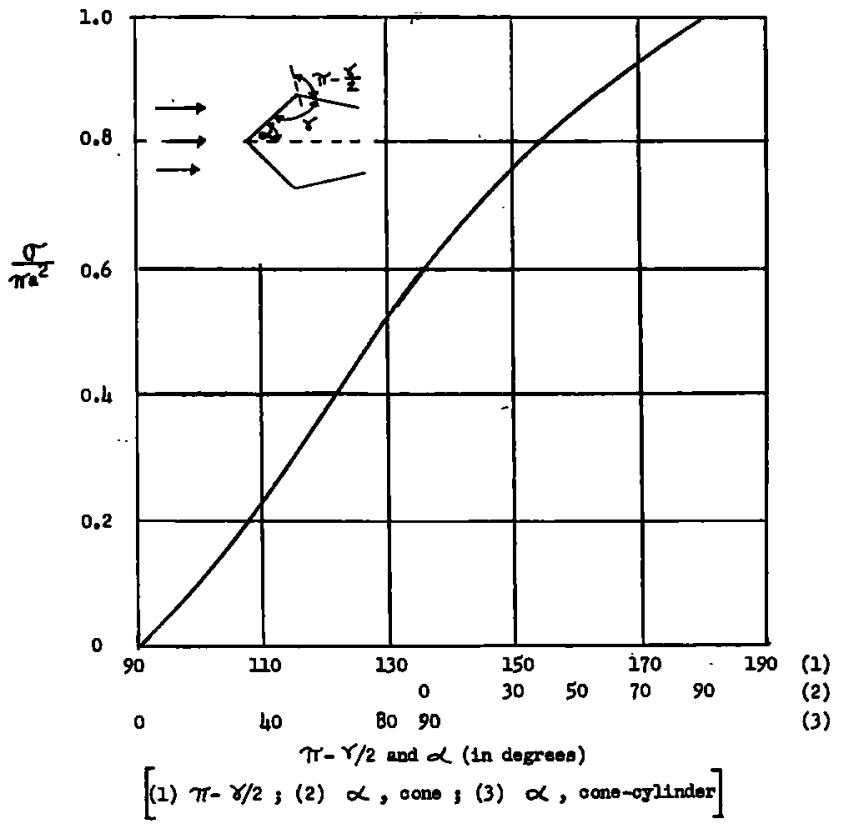

Fig. 3.13. Nose-on cross-section of circular wedge.

A similar technique of decomposition into straight segments was employed by Artmann ${ }^{15}$ ) in his solution of the problem of 
diffraction by a thick half-plane. He considered a half-plane of thickness $2 a$ capped by a half-cylinder of radius $a$, as in fig. 3.14. For $k a \gg 1$ and incidence as indicated he decomposed the cylindrical portion into a regular $N$-gon of length $L \gg \lambda$. Then by considering the conditions under which the rays striking near the apex $S$ are
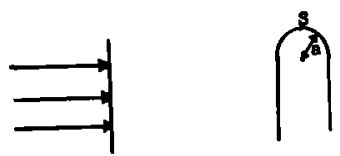

Fig. 3.14. Thick half plane.

diffracted on to the next side of the $N$-gon he determined the size of the penumbra region and hence the shift in the diffraction pattern as compared with the diffraction pattern of a completely black

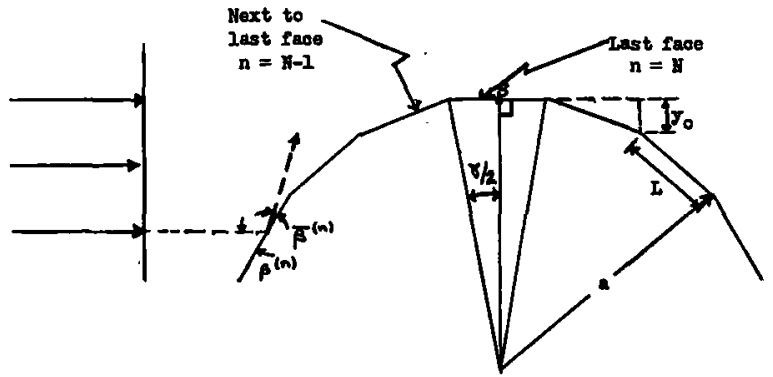

Fig. 3.15. $N$-gon geometry.

screen of like form. In order for rays diffracted from one polygonal face to have any effect on the next face, the following inequality must hold:

$$
k L\left|\cos \beta^{(n)}-\cos \bar{\beta}^{(n)}\right| \lesssim 2 \pi .
$$

The quantities involved are shown in figure 3.15. To measure the penumbra width, or in this approximation the number and length of the polygonal sides that have any effect on succeeding sides, Artmann proceeds as follows. First he restricts the sides so that the only ones that affect the next one are the last and next last, where the last side contains the apex and-naturally the next to last side immediately precedes it on the lit side (see fig. 3.15). In oxder 
that this be true

$$
k L\left|\cos \beta^{(N-1)}-\cos \bar{\beta}^{(N-1)}\right| \approx 2 \pi .
$$

From fig. 3.15 we see that

$$
\beta^{(N-1)}=\gamma
$$

Since the next to last face $(n=N-1)$ is not affected by rays from the preceding face $(n=N-2)$,

$$
\bar{\beta}^{(N-1)}=0 .
$$

Substituting these values in (3-46) yields

$$
k L|\cos \gamma-1| \approx 2 \pi
$$

but

$$
\cos \gamma \approx 1-\frac{1}{2} \gamma^{2}
$$

hence

$$
\frac{1}{2} k L \gamma^{2} \approx 2 \pi .
$$

Again referring to fig. 3.15 we see that

but

$$
\sin \frac{1}{2} \gamma=L / 2 a
$$

$$
\sin \frac{1}{2} \gamma \approx \frac{1}{2} \gamma,
$$

hence

$$
L \approx \gamma a
$$

and

$$
k a \gamma^{3} \approx 4 \pi
$$

or

$$
\gamma \approx\left(\frac{4 \pi}{k a}\right)^{t}
$$

Once more referring to fig. 3.15 we see that

$$
y_{0}=L \sin \gamma,
$$

or employing the above results

$$
y_{0} \approx \gamma^{2} a \approx\left(\frac{4 \pi}{k a}\right)^{2} a .
$$

Hence, according to Artmann, the diffraction pattern of the thick screen is displaced by this distance $(4 \pi / k a)^{*} a$ perpendicular to the 
direction of incidence as compared with the diffraction pattern of a completely black screen.

The above small wavelength approximations assist us in obtaining approximate far zone cross sections for many bodies of revolution. We must now describe what can be done to obtain results in the resonance region.

$\S 4$. The resonance region. To obtain answers for prolate spheroids when the radius of curvature at the tip $\left(b^{2} / a\right)$ is small with respect to the wavelength and simultaneously when the wavelength is small with respect to the broadside radii of curvature $b$ and $a^{2} / b$, we must use another type of approximation. A point in electromagnetics is physically a region where all radii of curvature are small with respect to the wavelength. Thus the thin prolate spheroid looks very much like an ogive.

The approximate theory used by Belkina for thin spheroids, which she compares with her exact answers ${ }^{18}$ ), and that used by Peters ${ }^{19}$ ) for thin ogives, as one might expect, are for the problem under consideration almost equivalent. Belkina's approximate theory is a special case of Peters' more general considerations. However, she obtains physical information from exact theory, not obtained by Peters as to when the approximation is valid for spheroids.

For axially symmetric transmission, scattering from infinite cones is extremely small in all directions except the specular direction. Local analysis near the front tip and in the penumbra region for thin prolate spheroids or ogives (since the reradiation is tangent to the path) provides no big scattering effect except in the forward direction. A good portion of energy is guided towards the rear point and again there occurs, primarily; a reflection back: The back flow of energy coming from the rear tip is again primarily in the forward direction (flow towards the front tip), which is in the direction back towards where it originally came from. Thus the back-scattering near nose-on cross-section of an ogive looks as if it is primarily due to the tip in the rear: This has been experimentally checked by Peters ${ }^{19}$ ).

This suggested to Peters and Belkina that the thin body should act like a travelling wave antenna. Peters derives the results for certain ogives and finds the cross-section for such an antenina 
(both monostatic and bistatic) for aspects out to $40^{\circ}$ off nose-on. The theory would fail exactly nose-on, but provides excellent results for near nose-on aspects.

To illustrate the theory we shall concentrate on a specific example, the thin prolate spheroid with $\boldsymbol{E}$ polarized field incident. The radar cross-section of a long thin body is given by

$$
\sigma=\frac{\gamma^{2} \lambda^{2}}{\pi Q^{2}}\left\{\frac{\sin \theta}{1-p \cos \theta} \sin \left[\frac{k L}{2 p}(1-p \cos \theta)\right]\right\}^{2}=\frac{\gamma^{2} \lambda^{2}}{\pi Q^{2}}[f(\theta)]^{2}
$$

where $Q$ is given by

$$
\begin{aligned}
& Q=-\left(2 / p^{2}\right)+\frac{\operatorname{Cin}[(k L / p)(1+p)]-\operatorname{Cin}[(k L / p)(1-p)]}{p^{3}}+ \\
& +\frac{1}{2 p^{3}}\{(p-1) \cos [(k L / p)(1+p)]+(p+1) \cos [(k L / p)(1-p)]+ \\
& \left.+\left(p^{2}-1\right) \frac{k L}{p}(\operatorname{Si}[(k L / p)(1+p)]-\operatorname{Si}[(k L / p)(1-p)])\right\}
\end{aligned}
$$

with Cin $(x)$ being the modified cosine integral of argument $x$ and $\mathrm{Si}=$ the sine integral. We see that there are three parameters besides the wavelength, which serve to describe the body. They are the voltage reflection coefficient $\gamma$, the relative phase velocity $p$ and the length $L$.

Voltage reflection coefficients of thick ogives and thin rods have been experimentally determined by Peters, who found that for a fairly thick ogive the reflection coefficient is about 0.7. For thin rods Peters found that the voltage reflection coefficient is about $\frac{1}{3}$. Physical reasoning indicates that the thin prolate spheroid, near nose-on, should be compared with a thin rod rather than an ogive, and hence for a thin prolate spheroid we use a voltage reflection coefficient of $\frac{1}{3}$. However, as $\theta$ increases from zero (the nose-on aspect), the point at which the travelling wave is reflected may be expected to move around the body and in this case will cause it to enter a region of larger radius of curvature. Thus, we would expect the voltage reflection coefficient to increase to 1 as the aspect goes to broadside. The actual values used in the graph (fig. 4.1) are as given in the following table:

\begin{tabular}{|c|c|c|c|}
\hline$\theta$ & $0^{\circ}-40^{\circ}$ & $40^{\circ}-60^{\circ}$ & $60^{\circ}-75^{\circ}$ \\
\hline$\gamma$ & 0.33 & 0.7 & 1.0 \\
\hline
\end{tabular}


The relative phase velocity $p$ is defined as the ratio of the length of the body to that of the current path on the body. For this case it turns out that $p=0.985$.

As the angle of incidence is increased from zero, a point is ultimately reached at which the travelling wave theory breaks down and the analogy with a thin wire is no longer possible. To deal with such values of $\theta$ (i.e., near broadside incidence) an alternative model is required. In this case the body is likened to a thick cylinder; the thick cylinder results are displayed for aspects in the range $\theta=60^{\circ}$ to $\theta=90^{\circ}$ in fig. 4.1. The thick cylinder results are obtained from ${ }^{20}$ ). The excellent, but as yet unpublished, experimental results of J. Lotsof of the Cornell Aeronautical Laboratory are included in fig. 4.1 for the purpose of comparison. Indeed, it was the existence of these experimental data which dictated the choice of the dimensions of the spheroid to be used in this illustrative example.

Before terminating this discussion of travelling wave theory, a few words about the $\boldsymbol{H}$ polarization case for the same prolate spheroid are in order. At near nose-on incidence we should expect the same current to be induced, and thus the same cross-section. However, with increasing $\theta$, the spiralling of the current may be expected to lead to an appreciable reduction in the cross-section; this has been confirmed by the above-mentioned experiments.

Now we shall turn out attention to the problem of estimating the nose-on scattering cross-section of thin finite cones for all values of $\left.k a^{*}\right)$. We need the approximate behaviour in the resonance region as we have already presented small and large wavelength approximations. This is obtained by assuming that the base is still the dominant feature as the resonance region is entered from the small wavelength side. The resonance maximum of the ring singularity would approximate, in both position and amplitude, the last large maximum of the cone. Since in any physically realizable situation the edge of the base of a cone will have a non-zero radius of curvature $b(b \ll \lambda)$, the only difference between it and a wire loop (wire radius $\ll \lambda$ ) relative to incident electromagnetic energy is that currents can exist "inside" the loop, but not "inside" the base of the cone.

When one looks at the axially symmetric cross-section of a ring

*) The quantity $a$ denotes the radius of the base of the cone as usual $k=2 \pi / \lambda$. 
as a function of wavelength, one finds that there are no minima. This, then, allows one to predict that the contribution of the inner edge is negligible in comparison to the outer edge when the wavelength is equal to the order of the loop radius but greater then the wire radius. (If there were non-negligible contributions from both the outer and inner edges, then at some wavelengths they would add

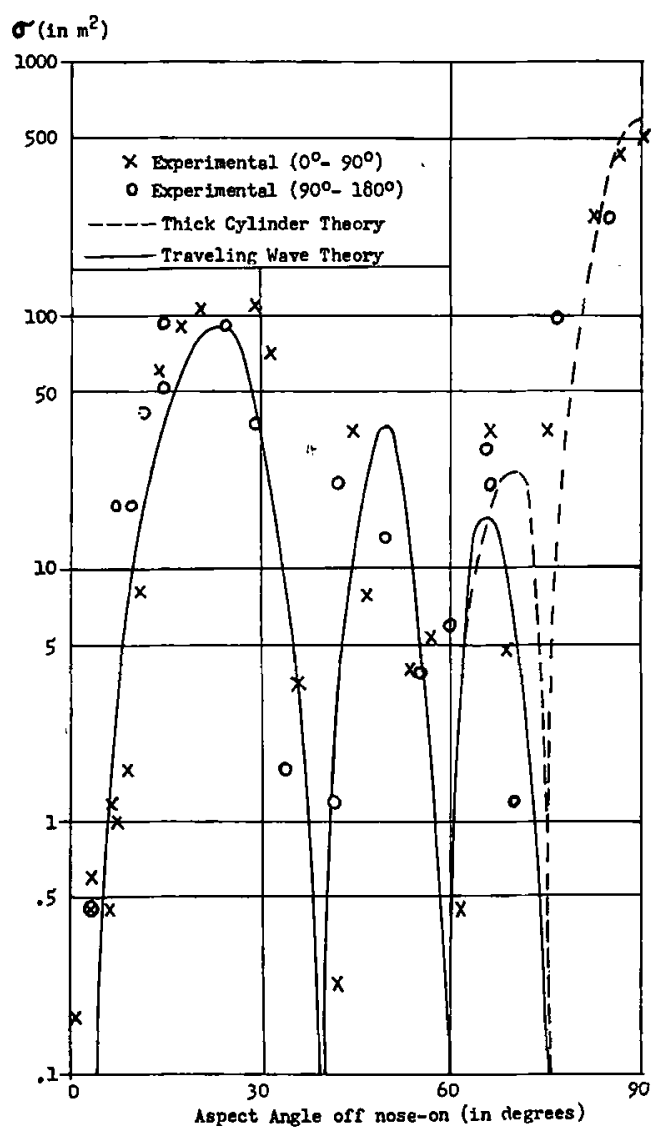

Fig. 4.1. Monostatic radar cross-section of a $10: 1$ prolate spheroid which is $43 \mathrm{~cm}=4 \lambda$ in length.

in phase and at some wavelengths they would add out of phase. But there are no noticeable minima in this region!) Thus the crosssection of a loop here looks like a Rayleigh side-type answer, depending only on the loop radius, but not on the wire radius. This, 
then, gives added justification for using an analogy between the conical base and the wire loop. Kouyoumjian's variational results ${ }^{21}$ ) and Weston's exact results ${ }^{22}$ ) for wire loops in the resonance region can then be utilized. Their results (as a function of wire radius and loop radius) indicate that the resonant peak is

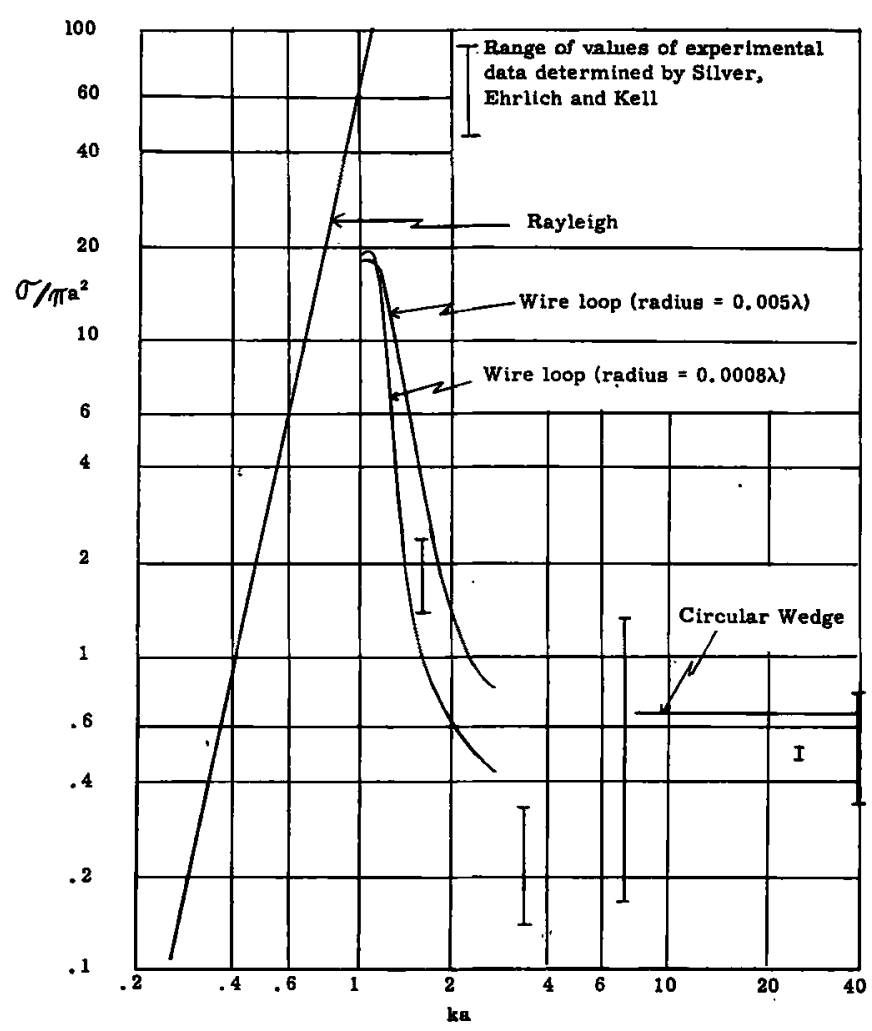

Fig. 4.2. Nose-on cross-section of thin finite cones $\left(\alpha=7.5^{\circ}\right)$.

fairly insensitive to changes in wire radius, but that as the wavelength decreases the wire radius becomes important. However, when the wavelength decreases, we use the wedge approximation. There may be a region on the small wavelength side of the loop maximum where other maxima, smaller in amplitude, can occur. These lesser extrema are essentially averaged in this approximation.

On the Rayleigh side we find that the Rayleigh line, which is an upper bound on the cross-section, lies so close to the ring maximum (in fact may intersect the ring curve before the maximum) that-the 
existence of any maxima greater than the ring maxima on the Rayleigh side is precluded. This is illustrated in fig. 4.2, where the experimental results of S. Silver of the University of California, R. Kell of the Cornell Aeronautical Laboratory and M. Ehrlich of the Microwave Radiation Company have been included for the purpose of comparison.

In order to obtain off axis finite cone results and to check our assumptions concerning the different reflection coefficients at the two ends, we have compared the off axis results for the cone with the travelling wave antenna result. We have added the Kirchhoff disc contribution to the back-scattering near rear-on answers. These

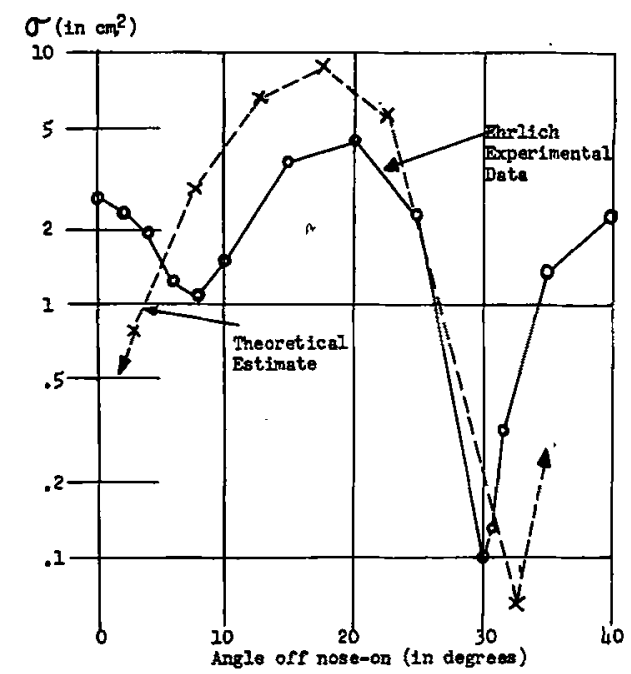

Fig. 4.3. Radar cross-section of a finite cone. A comparison between theory and experiment $\mathrm{I}$.

theoretical estimates are compared with the corresponding experimental data obtained by Ehrlich in figs. 4.3 and 4.4. We note that the null near the rear-on aspect is theoretically predicted to be too near to the $\theta=180^{\circ}$ aspect. This could have been anticipated since we know from the resonance discussion of the importance of the disc contribution.

By using approximations based on a creeping wave type picture we supplement the above theory for nose-on results where $f(\theta)=0$. For ogives of half angle $\alpha, \alpha \leqslant 20^{\circ}$, and $k a \geqslant 15(a=$ half maximum 
minor dimension) we obtain a nose on result of

$$
\sigma(0)=\frac{\lambda^{2} \tan ^{4} \alpha}{16 \pi}+\frac{1}{2} a \lambda
$$

When the creeping wave contribution is neligible, the $\frac{1}{2} a \lambda$ augmentation disappears. This occurs for thick ogives. The above formula holds for all ogive experiments analyzed to date within a factor of two. A feeling for when to drop out the term $\frac{1}{2} a \lambda$ can be obtained from known results for spheres.

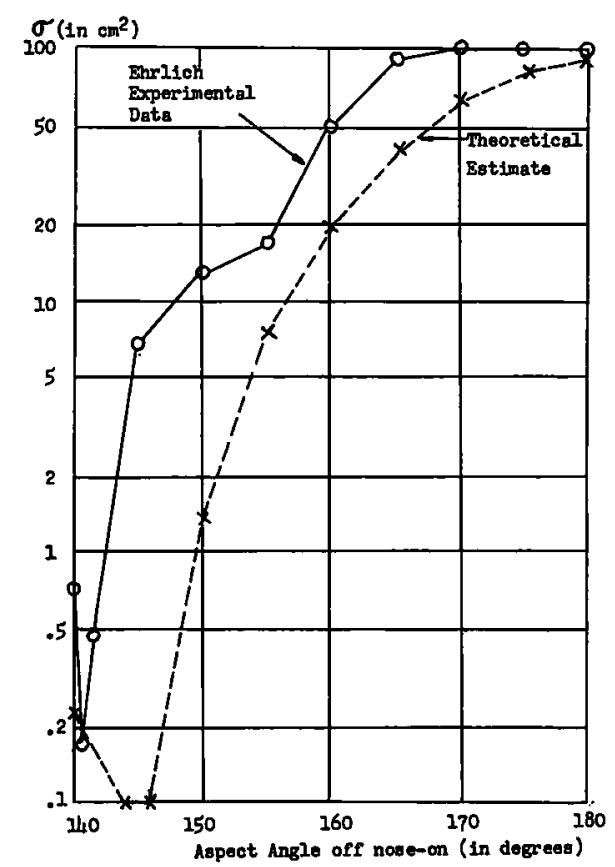

Fig. 4.4. Radar cross-section of a finite cone. A comparison between theory and experiment II.

The reader is now in a position to fill in roughly the complete cross-section curves for ogives and spheroids.

Acknowledgment. I gratefully acknowledge the assistance of H. Brysk, R. F. Goodrich, J. W. Crispin, R. E. Kleinman, D. M. Raybin and C. E. Schensted in the writing up of these remarks. Almost all my efforts associated with slots, directly and by reciprocity, were sponsored by the Hughes Aircraft Company. 
I specifically thank M. D. Adcock, R. Bickmore, R. Hansen and $M$. Chernin for many interesting discussions. I also thank $S$. Chapman, G. Graham, R. Kell and J. Lotsof of Cornell Aeronautical Laboratory, Inc., for allowing me to use their experimental results and for supporting my effort on the prolate spheroid scattering calculations. Professor Silver of the University of California and Professor Sinclair of the University of Toronto have in many cases helped me to crystallize my ideas and encouraged me through many stimulating discussions.

The speculations in this paper are mine and the above scientists are in no way responsible for my inaccuracies or misguesses.

Received 12th June, 1958.

\section{REFERENCES}

1) Strutt, J. W., Baron Rayleigh, Phil. Mag. 44 (1897) 28.

2) Stratton, J. A., Electromagnetic Theory, McGraw-Hill, New York, 1941.

3) Fock, V. A., J. Phys. 10 (1946) 399.

4) Rice, S. O., Bell Syst. Tech. J. 33 (1954) 417.

5) Franz, W., Z. Naturforsch. ga (1954) 705.

6) Keller, J. B., Trans. Inst. Radio Engrs AP (1956) 312.

7) Franz, W., and K. Deppermann, Ann. Phys. Lpz. 10 (1952) 361.

8) Friedlander, F. G., Comm. Pure Appl. Math., 7 (1954) 705.

9) Wu, T. T., Phys. Rev., 104 (1956) 201.

10) Bailin, L. L., Trans. Instn Radio Engrs AP 3 (1955) 128.

11) Wetzel, L. and D. 'B. Brick, An Experimental Investigation of High-Frequency Current Distributions on Conducting Cylinders, Air Force Cambridge Research Center, Scientific Report No. 4, Dec. 12, 1955.

12) Fock, V. A., J. Phys. g (1945) 255.

13) Belkina, M. G. and L. A. Weinstein, Radiation Characteristics of Spherical Surface Antennas, Appearing in Diffraction of Electromagnetic Waves on Certain Bodies of Rotation, Moscow, 1957.

14) Logan, N. A., The Role of Fock Functions in the Theory of Diffraction by Convex Surfaces, Air Force Cambridge Research Center, Paper presented at the URSI meeting held May 22-25, 1957, in Washington, D.C.

15) Artmann, K., Z. Phys. 127 (1950) 468.

16) Siegel, K. M., H. A. Alperin, R. R. Bonkowski, J. W. Crispin, A. L. Maffett, C. E. Schensted and I. V. Schensted. J. Appl. Phys. 26 (1955) 297.

17) Oberhettinger, F., Commun. Pure Appl. Math. 7 (1954) 551.

18) Belkina, M. G., Radiation Characteristics of an Elongated Rotary Ellipsoid, Appearing in Diffraction of Electromagnetic Waves On Certain Bodies of Revolution, Moscow, 1957.

19) Peters, L., Trans. Instn Radio Engrs AP 6 (1958).

20) Mentzer, J. R., Scattering and Diffraction of Radio Waves, Pergamon Press, 1955.

21) Kou youmjian, Appl. Sci. Res. B6 (1956) 165.

22) Weston, V. H., Solutions of the Toroidal Wave Equation and their Applications, University of Toronto, Thesis 1956. 